\title{
EPFIA: Extensible P2P Flows Identification Architecture
}

\author{
Bo Xu, Bing Li, Chao Hu, Guomin Zhang \\ Institute of Command Information System PLA University of Science and Technology, Nanjing, China \\ Email: xubo820@163.com, Libing781@163.com,Huchao1007@163.com, zhang_gmwn@163.com
}

Received August 2013

\begin{abstract}
The fundament of managing P2P traffic is identifying various P2P flows accurately. Although many P2P flows identification methods are presented nowadays, there are no ideas for either integrating these independent methods together or being extended fast to support new method. In this work, an extensible P2P flows identification architecture (EPFIA for short) is proposed. In order to identify many specific P2P flows, EPFIA uses many different identification methods simultaneously, and obtains the highest efficiency via adjusting their identification sequence. An online mechanism of renewing identification methods is designed, which can extend new identification method without compiling the whole program. Applying policy mechanism, identification methods can be updated, started and halted remotely. The experiment results of running the prototype system show us that EPFIA could effectively promote the performance of system and support online renew P2P identification methods and manage them remotely.
\end{abstract}

Keywords: P2P; Flow Identification; Architecture; Policy Schedule

\section{Introduction}

$\mathrm{P} 2 \mathrm{P}$ technology is one of the most exciting areas of Internet, which uses the idle resources in network edge, and makes Internet computing pattern from centralized to edge. Recent studies show that more than $60 \%$ of Internet traffic is attributed to P2P applications, of which BitTorrent and eDonkey traffic is of $80 \%$ of the total P2P traffic [1].

In this paper we define flow as bidirectional flow determined by 5 -tuple (source and destination address, source and destination port, and transport layer protocol) and 64 seconds timeout is adopted [2]. Generally P2P traffic identification methods can be divided into 4 types: transport layer port based identification method $\left(f_{\text {port }}\right.$ for short), application layer signature based identification method ( $\boldsymbol{f}_{\text {sig }}$ for short), transport layer behavior based heuristic identification method ( $f_{\text {behavior }}$ for short), and machine learning based identification method $\left(f_{M L}\right.$ for short). After the invalidation of $\boldsymbol{f}_{\text {port }}$ [3], researchers pay more attention to $f_{\text {sig, }}$, which uses the packet payload signature to identify the application accurately [3-6]. $\boldsymbol{f}_{\text {behavior }}$ uses flow attributes, statistics and behavior feature to identify P2P application[7,8]. $\boldsymbol{f}_{M L}$ constructs classifier with the flow characteristics of different protocol, and then use the classifier to identify the applications of unknown flows $[9,10]$. Besides, there are also crawlers based identification methods, etc. As the development of new P2P applications, new identification methods will keep on emerging. Thus, current P2P flow identification methods have the following problems: firstly, identifying one kind of P2P applications need one kind of device, this will be too hard to deploy system that can identify many kinds of P2P applications; secondly, the structure of identification system is inflexible, and it needs recompile the system software (even redesign system architecture) to extend or modify some new identification methods; thirdly, updating or maintaining identification system is very difficult.

We propose EPFIA to assemble many P2P traffic identification methods. EPFIA has the following features: improving identification efficiency via optimizing the sequence of identifying different P2P applications; designing a mechanism to update identification methods online, which can extend the P2P flow identification function without recompile the software; using policy mechanism, the update, start, and stop identification method can be carried out remotely.

Moore and Papagiannaki used port numbers and application layer payload to identify P2P flow [6]. They sorted the nine identification methods based on the ascending order of implementation complexity so as to identify the application of each flow, and pay no attention to the impact of the component of traffic on identification system performance. Most of current works focus on P2P application features, and studied the accuracy and efficiency of identification methods, they cannot extend new identification method under existing architecture, and cannot identify unknown traffic.

The remainder of this paper is organized as follows: Section 2 proposes the architecture of EPFIA and dis- 
cusses the impact of identification methods running sequence on system performance. Section 3 presents an online identification methods updating mechanism. Section 4 studies the method of using policy mechanism to maintain and update identification system. Section 5 validates the feasibility of EPFIA and tests its performance. Finally, Section 6 summarizes the whole paper.

\section{Design of EPFIA}

\subsection{Design Principle}

The main work process of EPFIA is as follows. Firstly, the packet capturer module is deployed at the bottom of EPFIA, which captures packets from the physical link, and filters packets that do not use TCP/UDP (Since P2P application usually using TCP/UDP transport protocol), and then submits packets to system identification modules in uniform format and style. In the paper, we use identification module denote the concrete implement of identification method. Using the uniform format so that it has packet information needed by any identification module, and using the uniform style because some identification methods only need one packet in a flow while others need a sequence of successive packets. Besides, the uniform format also makes all identification modules can work with the same packet capturer module. Since capturing packets from physical link consumes a large amount of system resources, it is of great important to use a uniform packet capturer module.

EPFIA handles packet based on flow state, which can be classified into two kinds: known and unknown. Known means a flow belongs to a kind of P2P or non-P2P application that has been identified, and it is of no need to identify it any more. While unknown means we need the identification module to handle the packet. Suppose $\Lambda$ is the packet information, and its format is shown in Figure 1.

The flow identifier $\boldsymbol{\Lambda}_{\text {flowID }}$ is calculated using function CreateHash based on the first 5 field in Figure 1, which can be used to determine which flow the packet belongs to, and then determine whether the packet belongs to unknown.

Finally, the unknown packet should be handled by many P2P identification modules, and change the flow state to be known. During this process, we can use $\boldsymbol{f}_{\text {port }}$ to identify non-P2P flow based on $\boldsymbol{\Lambda}_{\text {srcPort }}$ and $\boldsymbol{\Lambda}_{\text {dstPort }}$, and use $\boldsymbol{f}_{\text {sig }}$ to identify P2P flow that has application layer sig-

\begin{tabular}{|c|c|c|c|}
\hline $\begin{array}{c}\text { source IP address } \\
\text { srcIP }\end{array}$ & $\begin{array}{c}\text { source port } \\
\text { srcPort }\end{array}$ & $\begin{array}{c}\text { destination IP address } \\
\text { dstIP }\end{array}$ & $\begin{array}{c}\text { destination port } \\
\text { dstPort }\end{array}$ \\
\hline $\begin{array}{c}\text { transport protocol } \\
\text { proto }\end{array}$ & $\begin{array}{c}\text { packet size } \\
\text { size }\end{array}$ & arrive time & flow identifier \\
time & flowID \\
\hline \multicolumn{4}{|c|}{ application layer payload: payload } \\
\hline
\end{tabular}

Figure 1. Format of packet information. nature. For other unknown flows, we should design corresponding identification method to identify them.

To achieve the goal of identifying many P2P applications in a system, we need to run many $\mathrm{P} 2 \mathrm{P}$ identification modules simultaneously, update these modules frequently, and manage these modules remotely. As an extensible architecture for identifying $\mathrm{P} 2 \mathrm{P}$ applications, we should focus on the following questions when designing EPFIA:

1. Whether these P2P flow identification modules should be run randomly or in some fixed sequence? If there are some relationships between efficiency and identification sequence, what are the relationships?

2. Since new identification methods keep on emerging, can we extend some new identification methods conveniently without recompile the existing program?

3. If we want to remotely update, start or stop a P2P identification module running at somewhere in the network, is there a good implementation mechanism?

\subsection{Best Running Sequence of Identification Modules}

EPFIA should call many specific identification modules so as to implement the identification of unknown flow. By changing the running sequence of many different identification modules, we can improve the whole working efficiency.

Suppose $\mathrm{p}(\mathrm{x})$ represents the computation cost of identification module $\mathrm{x}$ when identifying a flow, and $\boldsymbol{f}_{\text {port }}$ cost is $\mathrm{p}\left(\mathrm{x}_{1}\right), \boldsymbol{f}_{\text {sig }}$ cost is $\mathrm{p}\left(\mathrm{x}_{2}\right), \boldsymbol{f}_{\text {behavior }}$ cost is $\mathrm{p}\left(\mathrm{x}_{3}\right), \boldsymbol{f}_{\boldsymbol{M L}}$ cost is $\mathrm{p}\left(\mathrm{x}_{4}\right)$. Based on the identification principle we know the following inequation hold on:

$$
\mathrm{p}\left(\mathrm{x}_{1}\right)<\mathrm{p}\left(\mathrm{x}_{2}\right)<\mathrm{p}\left(\mathrm{x}_{3}\right)<\mathrm{p}\left(\mathrm{x}_{4}\right)
$$

During time $\mathrm{T}$, if there are $\mathrm{F}$ flows, and the percentage of flows that identified by identification module $\mathrm{x}$ is $\mathbf{f}_{\mathbf{x}}$, we give the following definitions:

Definition 1: Identification Module Running Sequence (Identification Sequence in short). Identification Sequence represents the set of identification modules and their running sequence. If we use $R_{n}$ to represent the Identification Sequence of $\mathrm{n}$ different modules, then $\mathrm{R}_{\mathrm{n}}=\left\{r_{l} \rightarrow \ldots \rightarrow r_{i} \rightarrow \ldots \rightarrow r_{n}\right\}$, in which $r_{i}$ represents the ith running modules.

Definition 2: System Identification Cost (Cost in short): The cost of identification sequence $R_{n}$ when identifying F flows in time T. We use POWER $\left(\mathrm{R}_{\mathrm{n}}\right)_{\mathrm{T}}$ represent Cost.

Definition 3: Priority Relationship P of Identification Modules (Priority for short). For any two identification modules in $R_{n}, P$ represents the running sequence that makes the Cost smaller. Suppose the Identification Sequence

$$
\begin{array}{r}
\mathrm{R}_{\mathrm{n}}=\left\{r_{l} \rightarrow \ldots \rightarrow x \rightarrow \ldots \rightarrow y \rightarrow \ldots \rightarrow r_{n}\right\}, \\
\mathrm{R}_{\mathrm{n}}=\left\{r_{l} \rightarrow \ldots \rightarrow y \rightarrow \ldots \rightarrow x \rightarrow \ldots \rightarrow r_{n}\right\},
\end{array}
$$


if and only if POWER $\left(\mathrm{R}_{\mathrm{n}}{ }^{\prime}\right)_{\mathrm{T}}<\operatorname{POWER}\left(\mathrm{R}_{\mathrm{n}}\right)_{\mathrm{T}}$, we say $\mathrm{y}$ and $x$ have Priority $\mathbf{P}$, and note it as $y \mathbf{P x}$.

Definition 4: Running Efficiency of Identification Module x (Running Efficiency for short). The ratio between the number of flows identified by $\mathrm{x}$ and the computation cost of $\mathrm{x}$ in time $\mathrm{T}$ is defined as Running Efficiency of $x$, which is represented as $E(x)=f_{x} * F / p(x)$.

Suppose $\mathrm{F}$ flows belong to $\mathrm{m}$ applications, and there are $\mathrm{n}$ identification modules that can identify each flow accurately. These modules have Priority $\mathbf{P}$ and we can adjust their running sequence randomly, then we have the following lemma.

Lemma 1: There is a best Identification Sequence $R_{n}$ that makes the POWER $\left(\mathrm{R}_{\mathrm{n}}\right)_{\mathrm{T}}$ minimum.

Proof: For the $\mathrm{n}$ identification modules, there is a sequence $\mathrm{R}_{\mathrm{n}}^{\prime}$ that $\forall x \forall y\left(x, y \in R_{n}^{\prime} \rightarrow x P y\right)$. If we exchange any two identification modules in $\mathrm{R}_{\mathrm{n}}$ and form $\mathrm{R}_{\mathrm{n}}$, from definition 3 we know $\operatorname{POWER}\left(\mathrm{R}_{\mathrm{n}}\right)_{\mathrm{T}}>$ $\operatorname{POWER}\left(R_{n}\right)_{T}$. That is, there is a best Identification Sequence $R_{n}$, in which any two identification modules have Priority $\mathbf{P}$, so that POWER $\left(R_{n}\right)_{T}$ is minimized.

Theorem 1: Identification modules that have high Running Efficiency have high Priority, that is $\forall x \forall y\left(x, y \in R_{n} \wedge E(x)>E(y) \rightarrow x P y\right)$.

Proof: Suppose $\mathrm{E}(\mathrm{x})<\mathrm{E}(\mathrm{y}), \mathrm{x}$ and $\mathrm{y}$ have the Priority P. Since the Running Efficiency of $y$ is higher than that of $x$, by running $y$ first we can reduce the number of unknown flows, and thus reduce the system identification cost, which conflicts with the assumption that the Priority of $x$ is higher than that of $y$. Thus, when running more than one identification module, we should adjust their running sequence according to their Running Efficiency so as to reduce Cost.

\subsection{Architecture of EPFIA}

Suppose $\mathrm{p}\left(\mathrm{x}_{2}\right)=\mathrm{m}_{2} \mathrm{p}\left(\mathrm{x}_{1}\right), \mathrm{p}\left(\mathrm{x}_{3}\right)=\mathrm{m}_{3} \mathrm{p}\left(\mathrm{x}_{1}\right), \mathrm{p}\left(\mathrm{x}_{4}\right)=\mathrm{m}_{4} \mathrm{p}\left(\mathrm{x}_{1}\right)$, then

$$
1<\mathrm{m}_{2}<\mathrm{m}_{3}<\mathrm{m}_{4}
$$

Non-P2P traffic accounts for about $30 \%$ of the total Internet traffic, and most of them use fixed ports to communicate with each other [1], thus we can use port number to identify them. The traffic of BitTorrent and eDonkey takes about $80 \%$ of total P2P traffic, and more than $73 \%$ and $83 \%$ of them do not encrypt their payload, thus we can use application layer signature to identify them. Other types of P2P application flow have no port and application layer payload feature, and thus we should design specific identification methods for them. Take the former discussion into consideration, the efficiency of $\boldsymbol{f}_{\text {port }}$ is $\mathrm{E}\left(\mathrm{x}_{1}\right) \approx 0.3^{*} \mathrm{~F} / \mathrm{p}\left(\mathrm{x}_{1}\right)$, and the efficiency of $\boldsymbol{f}_{\text {sig }}$ is $\mathrm{E}\left(\mathrm{x}_{2}\right) \approx 0.41 * \mathrm{~F} / \mathrm{p}\left(\mathrm{x}_{2}\right)=\frac{0.41 \times F}{m_{2} \times p\left(x_{1}\right)}$, and the efficiency of

$$
\boldsymbol{f}_{\text {behavior }}+\boldsymbol{f}_{M L} \text { is } \mathrm{E}\left(\mathrm{x}_{3}+\mathrm{x}_{4}\right) \approx \frac{0.29 \times F}{p\left(x_{3}\right)+p\left(x_{4}\right)}>\frac{0.29 \times F}{m_{4} \times p\left(x_{1}\right)}
$$

Each identification module identifies the application of flow according to $\boldsymbol{\Lambda}$, and we can use the number of packet to estimate the computation cost of a special identification module. Based on the principle of different identification methods, we know $\mathrm{m}_{4} \geq \mathrm{m}_{3} \geq 3$, and thus

$$
\mathrm{E}\left(\mathrm{x}_{1}\right)>\mathrm{E}\left(\mathrm{x}_{2}\right)>\mathrm{E}\left(\mathrm{x}_{3}+\mathrm{x}_{4}\right)
$$

As a result, we can get the best efficiency by using the following Identification Sequence: firstly using $\boldsymbol{f}_{\text {port }}$ to identify non-P2P flows, and then using $\boldsymbol{f}_{\text {sig }}$ to identify un-encrypted P2P flows, finally using other special methods to identify the remainder P2P flows.

Observed that the identification is based on flow, and as the process of identification, the percentage of identified flows will keep on increase. If there are $\mathrm{M}$ packets in a new flow $\boldsymbol{f}_{\text {new }}$, and an identification module can identify the application of $\boldsymbol{f}_{\text {new }}$ based on the first $\mathrm{n}$ packets, thus the remainder M-n packets will need not to be identified any more. Generally $\mathrm{M}>>\mathrm{n}$, we can increase the identification efficiency by identifying which flow the M-n packets belong to. Thus we design a flow differentiation module, which identifies whether a coming packet belongs to a known or an unknown flow, and we only handle packets that belong to unknown flows. Experiment results show that this method can filter about $98 \%$ packets, and thus decreasing the identification cost effectively.

Based on the upper discussion, we designed an extensible $\mathrm{P} 2 \mathrm{P}$ flow identification architecture, which is shown in Figure 2.

EPFIA uses flow identifier $\boldsymbol{\Lambda}_{\text {flowID }}$ as the address of Hash-Table item, and this address is also used as the first address of storing active flow information flow-key. flow-key $=$ \{source IP address srcIP, source port srcPort, destination IP address dstIP, destination port dstPort,

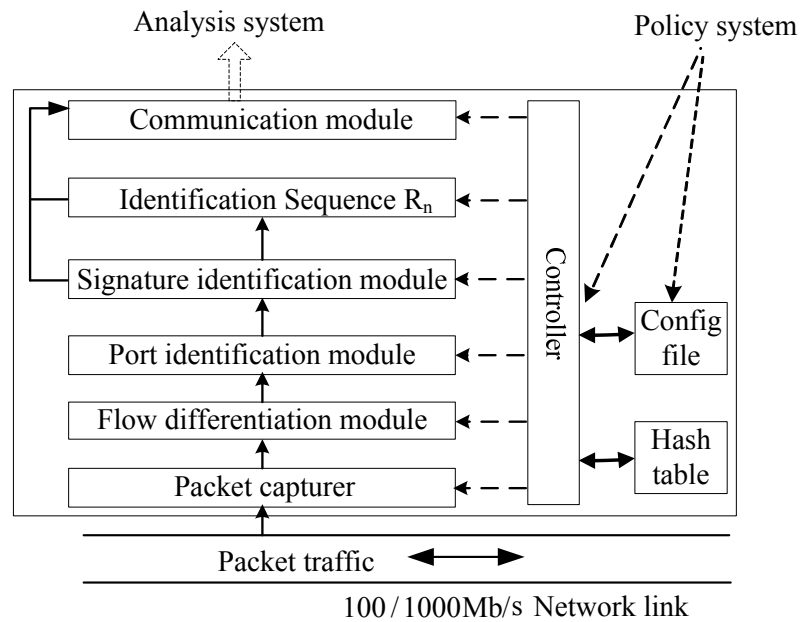

Figure 2. Extensible P2P flow identification architecture. 
transport protocol Proto, flow state State \}, in which State $\in\{-1,0, \mathrm{P} 2 \mathrm{P}$ ID $\}$, and " -1 " represents unknown flow, "0" represents non-P2P flow, and "P2P_ID" represents the $\mathrm{P} 2 \mathrm{P}$ application code defined by us. In addition, each flow has a counter, which is used to record the number of packets identified for unknown flows. When counter is larger than a threshold $\Phi$, we change the state of that corresponding flow to be a non-P2P flow. We also use $[\boldsymbol{P O R T}]_{\text {std }}$ to represent standard network service ports, and use $[S I G]_{P 2 P}$ to represent the set of specific P2P application signatures.

\section{Identification Program Online Updating Mechanism}

In order to add new identification module, maintain and update current module and adjust the running sequence of different modules conveniently, EPFIA uses the form of plug-in to manage different identification modules. EPFIA stores all identification modules in a plug-in database, and stores the running sequence $R_{n}$ in a policy database. Figure 3 shows the format of plug-in stored in policy database.

In which "Priority" is a number that defines the plugin's running sequence; and "P2P application code" denotes the $\mathrm{P} 2 \mathrm{P}$ application that this plug-in can identify; and controller uses "Store path" and "Plug-in name" to locate the plug-in, and use "Main control function" to run this identification module. Figure 4 shows the online updating mechanism of EPFIA.

During the initialization phase, the main control module accesses the policy database to get $R_{n}$, and then stores all plug-in information in the memory using list structure, which is sorted by priority. During the identification phase, the controller calls different identification modules to handle each packet according to the priority sequence.

EPFIA updates identification module by maintaining the plug-in database, and adds new identification module

\begin{tabular}{|l|l|l|l|l|}
\hline Plug-in name & Store path & Main control function & P2P application code & Priority \\
\hline
\end{tabular}

Figure 3. Format of plug-in stored in policy database.

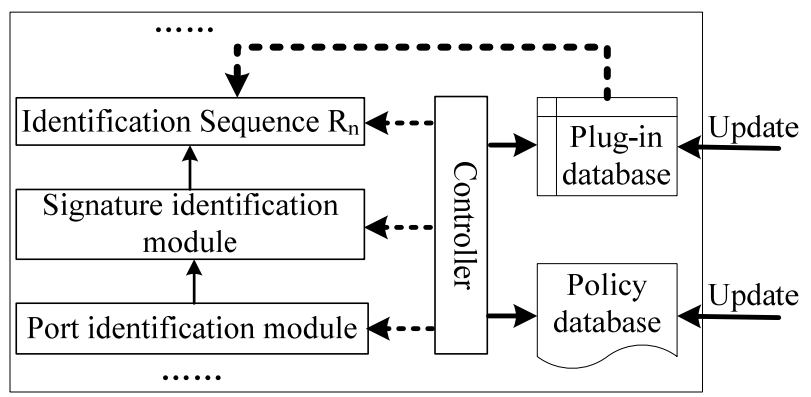

Figure 4. Online updating mechanism of EPFIA. as well as adjusts the running sequence of different identification modules by handling the list, thus it avoids recompiling the program to implements such functions.

\section{Policy Mechanism}

EPFIA uses policy mechanism to support updating, starting and stopping identification modules remotely. The policy is divided into two kinds: manually and automatically. The manual policies are setup by administrator, which include identification modules running sequence $\mathrm{R}_{\mathrm{n}}$ and parameters; automatic policies are generated using policy control language by EPFIA during the system running time, and they optimize the system efficiency by adjusting $R_{n}$ dynamically. Figure $\mathbf{5}$ shows the EPFIA policy mechanism.

In EPFIA, the controller provides two access interfaces: one is the plug-in database access interface FAP, and the other is the policy database access interface PAP. Administrators can access FAP and PAP through control center. The controller stores the received plug-in in the plug-in database, and stores the received policy in the policy database, and then updates $R_{n}$ according to the new policy.

According to Lemma 1 and Theorem 1, the running sequence of P2P flow identification modules can affect the efficiency of EPFIA. Though administrators can setup the running sequence of identification modules, it is very difficult to estimate the Running Efficiency of each identification module accurately and adjust their Priority immediately. EPFIA uses automatic policy and adjusts the module Priority dynamically. EPFIA determines the relative computation cost $\mathrm{m}_{\mathrm{x}}$ of program $\mathrm{x}$ according to the number of packets $\mathrm{x}$ needs to handle. $\mathrm{F}(\mathrm{x})_{\mathrm{T}}$ represents the number of flows $x$ identified during time $T$, and we use $\mathrm{E}(\mathrm{x})_{\mathrm{T}}=\mathrm{F}(\mathrm{x})_{\mathrm{T}} / \mathrm{m}_{\mathrm{x}}$ to represent the efficiency of $\mathrm{x}$ during time $\mathrm{T}$. The automatic control policy computes the current identification efficiency $\mathrm{E}(\mathrm{x})_{\text {current }}$ of $\mathrm{x}$ based on $\mathrm{E}(\mathrm{x})_{\mathrm{T}}$ and the old identification efficiency $\mathrm{E}(\mathrm{x})_{\text {old }}$.

$$
\mathrm{E}(\mathrm{x})_{\text {current }}=(1-\varepsilon) \cdot \mathrm{E}(\mathrm{x})_{\mathrm{T}}+\varepsilon \cdot \mathrm{E}(\mathrm{x})_{\text {old }}
$$

In Equation (4), $\varepsilon$ is a decimal fraction between 0 and 1 , which is used to control the sensitivity of efficiency

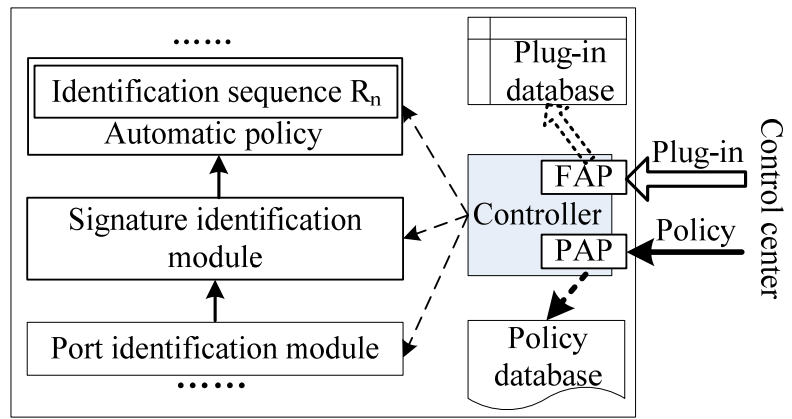

Figure 5. EPFIA policy mechanism. 
variation. The automatic control policy calculates the current identification efficiency $\mathrm{E}\left(\mathrm{x}_{\mathrm{i}}\right)_{\text {current }}$ of module $\mathrm{x}_{\mathrm{i}}(1$ $\leq \mathrm{I} \leq \mathrm{n})$ periodically, and determines the Priority according to $\mathrm{E}\left(\mathrm{x}_{\mathrm{i}}\right)_{\text {current }}$. Our experiment results show that the automatic control policy in EPFIA can adapt to the network traffic variation and increase the whole identification efficiency.

\section{Experiments and Analysis}

To validate the upper analysis, we developed the prototype system of EPFIA named P2P-Analyzer, which includes two P2P flow identification programs: one for UDP based Skype voice flow (Skype_Detection, SD), and the other for PPLive video flow (PPLive Detection, PD). We test the performance of P2P-Analyzer for a long while in the campus network of PLAUST, and following is the typical experiment result and its analysis.

\subsection{Experiment Environment and Method}

The experiment environment of P2P-Analyzer is shown in Figure 6. The campus network connects to the CERNET through $100 \mathrm{Mbps}$ fiber, and the experimental hosts have Intel Core $2 \mathrm{CPU}$ with $2.33 \mathrm{GHz}$ frequency, and 2 GB memory. In the campus network we install Skype, PPLive and BitTorrent client, which run randomly during the experiment. The running information such as communication begin time, number of communications and download file size are also recorded manually. The Tcpdump is running at the mirror port of the switch so as to capture the packet information with 50 bytes application layer payload. The P2P-Analyzer is also run to identify P2P flow automatically, and send the identified P2P flow information flow-keys to P2P-Looker to handle. $\mathrm{P} 2 \mathrm{P}-$ Looker is the software that can analyze multi-type $\mathrm{P} 2 \mathrm{P}$ flow information, so we can decrease the influence of analyzing on P2P-Analyzer performance. Besides, we also setup a control center so as to manage P2P-Analyzer remotely.

In order to compare and analyze the performance of

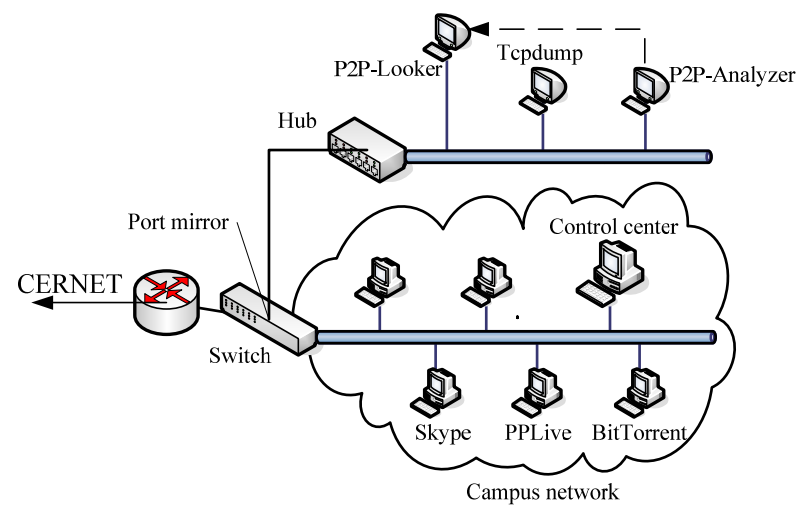

Figure 6. Experiment environment of P2P-Analyzer.
P2P-Analyzer, we firstly analyze the P2P flow information manually using data captured by Tcpdump, and use this result as the ground truth. Then compare it with the results identified by $\mathrm{P} 2 \mathrm{P}$-Analyzer. The counter is set in each module of P2P-Analyzer to record packets handled. For every 5 minutes the CPU usage of each module is calculated.

\subsection{Experiment Results and Analysis}

We analyze two typical experiment results carried out at different time. The packet information collected by Tcpdump is stored in two trace files, and the manual analysis result is shown in Table 1. Others include HTTP, FTP, DNS, Flash, FTP, ICMP, IMAP, MSN, POP, QQ, SMTP as well as unknown traffic, of which HTTP and Flash is more than $85 \%$.

In the first experiment, SD runs in the first two hours, and PD is called by policy in the last two hours. We setup $\Phi=10$ (the maximum number of packet that SD needs to identify Skype). The counter is outputted every hour so as to examine the performance of EPFIA. The result is shown in Table 2, from which we can see that the packet capturer filters $1 \%$ non TCP/UDP traffic, and the flow differentiation module filters $98 \%$ of the total packet, which decrease the load of identification modules dramatically. The number of unknown packet is decreased after calling PD. In addition, PD can identify PPLive flow before the number of packet reaches $\Phi$, and thus decreases the load of other identification modules further.

Figure 7 shows the CPU usage during the first experiment. In the first two hours when running $\mathrm{SD}$, the average CPU usage is $11.3 \%$, and in the last two hours when running $\mathrm{SD}$ and $\mathrm{PD}$ together, the average $\mathrm{CPU}$ usage is $10.1 \%$. From this we see that when adding new module, the usage of CPU can decrease because of the Running Efficiency of identification modules.

In the second experiment, we setup the relative computation load of PD and SD according to the number of

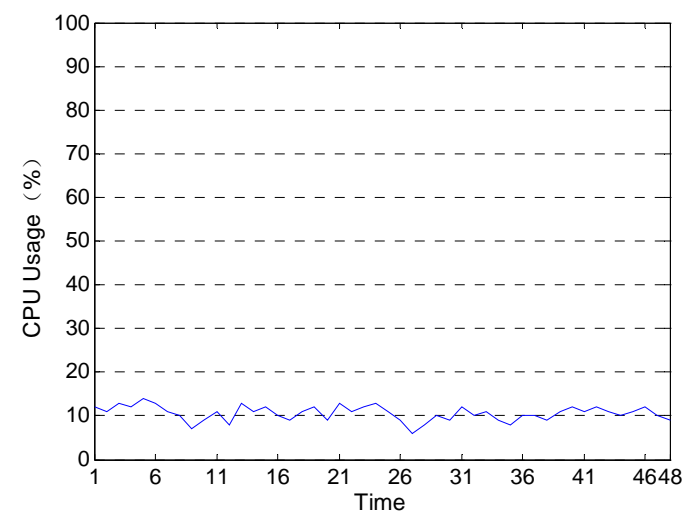

Figure 7. CPU usage information during the first experiment. 
Table 1. Manual analysis result of two traces.

\begin{tabular}{|c|c|c|c|c|c|c|c|c|c|c|c|c|}
\hline \multirow{2}{*}{ Traces } & \multirow{2}{*}{ Start } & \multirow{2}{*}{ Dur } & \multicolumn{5}{|c|}{ Flows } & \multicolumn{5}{|c|}{ Packets } \\
\hline & & & Total & Skype & $B T$ & PPLive & Others & Total & Skype & $B T$ & PPLive & Others \\
\hline $\mathrm{T} 1$ & $\begin{array}{c}\text { Mar20 } \\
08: 00\end{array}$ & $4 \mathrm{~h}$ & $99.3 \mathrm{~K}$ & $0.3 \mathrm{~K}$ & $51 \mathrm{~K}$ & $30 \mathrm{~K}$ & $18 \mathrm{~K}$ & $89 \mathrm{M}$ & $6.2 \mathrm{M}$ & $48.6 \mathrm{M}$ & $20.2 \mathrm{M}$ & $13.9 \mathrm{M}$ \\
\hline $\mathrm{T} 2$ & $\begin{array}{c}\text { Mar21 } \\
14: 00 \\
\end{array}$ & $5 \mathrm{~h}$ & $129.7 \mathrm{~K}$ & $0.7 \mathrm{~K}$ & $62 \mathrm{~K}$ & $44 \mathrm{~K}$ & $23 \mathrm{~K}$ & $113.6 \mathrm{M}$ & $13.7 \mathrm{M}$ & $51.7 \mathrm{M}$ & $29.8 \mathrm{M}$ & $18.3 \mathrm{M}$ \\
\hline
\end{tabular}

Table 2. Number of packets disposed by different modules in P2P-analyzer.

\begin{tabular}{|c|c|c|c|c|}
\hline \multirow{2}{*}{ Module name } & \multicolumn{2}{|c|}{ First two hours } & \multicolumn{2}{|c|}{ Last two hours } \\
\hline & $\begin{array}{c}\text { packet } \\
\text { number }\end{array}$ & percentage & $\begin{array}{c}\text { packet } \\
\text { number }\end{array}$ & percentage \\
\hline Packet capturer & $43 \mathrm{M}$ & - & $46 \mathrm{M}$ & - \\
\hline Flow differentiation module & $42.6 \mathrm{M}$ & $99 \%$ & $45.6 \mathrm{M}$ & $99.1 \%$ \\
\hline Port identification module & $379 \mathrm{~K}$ & $0.86 \%$ & $215 \mathrm{~K}$ & $0.46 \%$ \\
\hline Signature identification module & $371 \mathrm{~K}$ & $0.84 \%$ & $207 \mathrm{~K}$ & $0.44 \%$ \\
\hline PPLive_Detection(PD) & - & - & $185 \mathrm{~K}$ & $0.39 \%$ \\
\hline Skype_Detection(SD) & $351 \mathrm{~K}$ & $0.8 \%$ & $170 \mathrm{~K}$ & $0.36 \%$ \\
\hline Unknown packets & $350 \mathrm{~K}$ & $0.79 \%$ & $169 \mathrm{~K}$ & $0.35 \%$ \\
\hline
\end{tabular}

packets needed to identify a flow. That is $\mathrm{m}_{\mathrm{PD}}=4, \mathrm{~m}_{\mathrm{SD}}=$ 10. Using Equation (4) to calculate the identification efficiency of each module, and set $\varepsilon=0.5, \mathrm{E}(\mathrm{PD})_{\mathrm{old}}=$ $\mathrm{E}(\mathrm{SD})_{\text {old }}=0$. We use policy mechanism to call SD and PD one after the other, so as to test the impact of automatic policy on system efficiency.

By analyzing the traffic data further, we see that there are 174 Skype flows in the first hour, and 11264 PPLive flows. According to automatic policy mechanism, in the second hour, the sequence of SD and PD should be adjusted. Figure 8 shows the number of packets SD and PD handled per hour, which includes Skype, PPLive and other unknown application packets. From Figure 8 we can see that in the first hour the number of packets handled by SD is a little larger than that of PD. This phenomenon demonstrates that by adjusting the running sequence of SD and PD according to their identification efficiency, the number of packets handled by SD decreased dramatically, and the identification efficiency of P2P-Analyzer increased.

\section{Conclusion}

Taking the problems of identifying large number of P2P flows into consideration, in this paper we propose an extensible P2P flows identification architecture (EPFIA). In order to obtain the highest efficiency of EPFIA, many identification methods should be arranged following the optimizing sequence. An online mechanism of renewing identification methods is designed, which can extend new P2P identification method without recompiling the

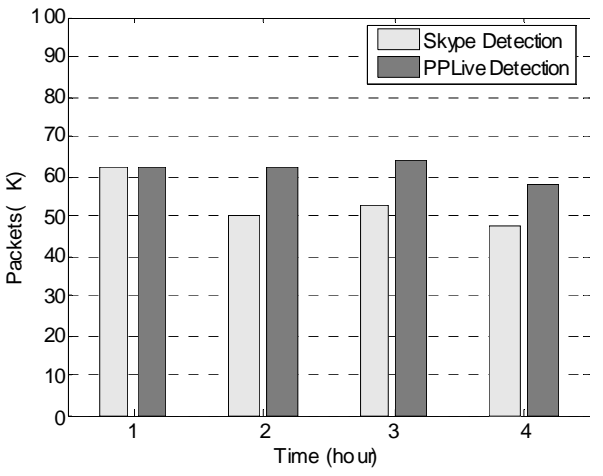

Figure 8. Number of packets SD and PD handled per hour.

whole program. Applying policy mechanism, identification modules can be updated, started and halted with policy remotely. The experiment results of running the prototype system show us that EPFIA could effectively promote the performance of system and support online renew $\mathrm{P} 2 \mathrm{P}$ identification methods and manage them remotely. In the further works, we will focus on deploying the system in large scale, and develop application systems based on P2P traffic analysis.

\section{REFERENCES}

[1] H. Schulze and K. Mochalski, "Internet Study 2008/2009," Technical Report, Ipoque GmbH, 2009.

[2] K. C. Claffy, "Internet Traffic Characterization," University of California, San Diego, 1994.

[3] T. Karagiannis and A. Broido, "Is P2P Dying or Just 
Hiding?" IEEE GLOBECOM 2004, Dallas, Texas, 29 November-3 December 2004, pp. 1532-1538.

[4] S. Sen and O. Spatscheck, "Accurate, Scalable In-Network Identification of P2P Traffic Using Application Signatures," $W W W 2005$, New York, USA, 17-22 May 2004, pp. 512-521.

[5] M. Roughan and S. Sen, "Class-of-Service Mapping for QoS: A Statistical Signature-Based Approach to IP Traffic Classification," IMC 2004, Taormina, Italy, 25-27 October 2004, pp. 135-148.

[6] A. Moore and K. Papagiannaki, "Toward the Accurate Identification of Network Applications," PAM 2005, Boston, USA, 31 March-1 April 2005.

[7] T. Karagiannis and A. Broido, "Transport Layer Identifi- cation of P2P Traffic," In IMC'04, Taormina, Italy, 25-27 October 2004, 14p.

[8] T. Karagiannis and K. Papagiannaki, "BLINK: Multilevel Traffic Classification in the Dark," SIGCOMM'05, Philadelphia, USA, 21-26 August 2005, 12p.

[9] L. Bernaille and R. Teixeira, "Early Application Identification," The 2nd ADETTI/ISCTE CoNEXT Conference, Lisboa, Portugal, December 2006.

[10] M. Crotti and M. Dusi, "Traffic Classification through Simple Statistical Fingerprinting," SIGCOMM Computer Communication Review, Vol. 37, No. 1, 2007, pp. 5-16. http://dx.doi.org/10.1145/1198255.1198257 